\title{
Comparison of the Effect on the Cuff Pressure of the Endotracheal Tubes Following Cuff Inflation with Air and With Nitrous Oxide - Oxygen during General Anaesthesia - A Prospective, Randomised Study
}

\author{
Dr. Manali Nadkarni ${ }^{1}$, Dr. R. D. Patel ${ }^{2}$, Dr.Sachin Navarange ${ }^{3}$ \\ I'Additional Professor, Department Of Anaesthesiology, Seth G. S. Medical College\& K.E.M.Hospital/ India) \\ 2(Professor, Department Of Anaesthesiology, Seth G. S. Medical College \& K.E.M. Hospital/ India) \\ ${ }_{3}^{3}$ (Ex Postgraduate Student, Department Of Anaesthesiology, Seth G.S.Medical College\&K.E.M.Hospital/ India)
}

\begin{abstract}
:
Background and aims: During general anaesthesia cuffed endotracheal tubes are used to provide airway seal and prevent aspiration. But it has been observed that use of nitrous oxide oxygen for ventilation during anaesthesia leads to rise in intracuff pressure which can be transmitted to the wall of trachea leading to complications. In this study we compared the effect on cuff pressure of Portex endotracheal tubes( high volume low pressure cuffs) and Armoured endotracheal tubes( low volume high pressure cuffs) when the cuffs were inflated with air and with nitrous oxide- oxygen mixture during general anaesthesia using nitrous oxide oxygen.
\end{abstract}

Method:After institutional ethics committee approval 180 patients aged 18 -60 years of ASA status I \& IIposted for elective surgeries were randomly allocated in four groups of 45 each. After taking consent and intravenous access general anaesthesia was given with endotracheal intubation. Group Al-Portexendotracheal tube cuff inflated with air. Group A2- Portex endotracheal tube cuff inflated with nitrous oxide- oxygen mixture. Group B1-Armoured endotracheal tube cuff inflated with air.Group B2-Armoured endotracheal tube cuff inflated with nitrous oxide- oxygen mixture. Controlled ventilation was done with nitrous oxide oxygen during the anaesthesia. Endotracheal tube cuff pressure was measured at inflation and at every 30 minutes interval till 90 minutes. Postoperatively patients were observed for any complications such as sore throat, difficulty in swallowing, hoarseness of voice, etc.

Results: It was observed that the intracuff pressure in both the high volume low pressure and the low volume high pressure cuffed endotracheal tubes rises with time when the cuffs are inflated with air. The rise was not significant when nitrous oxide - oxygen was used for inflating the cuff in both the groups. Regardless of the type of inflating medium used for cuff inflation the incidence of complications was high in low volume high pressure cuffed endotracheal tubes as compared to high volume low pressure cuffed endotracheal tubes.

Keywords: Air, cuff inflation, endotracheal tube cuff pressure, inflating agents, nitrous oxide- oxygen.

\section{Introduction}

Cuffed endotracheal tubes are used during anaesthesia as the most dreaded complication in a paralysed patient is aspiration. However the concerns over cuff to tracheal wall pressure and reports of aspiration past the low pressure cuffs have prompted changes in cuff design and introduction of cuff pressure regulating devices. The cuffs of the endotracheal tubes are of two types. They are high volume low pressure cuffs as in Portex endotracheal tubes and low pressure high volume cuffs as in armoured endotracheal tubes. The cuff when inflated exerts pressure on the wall of the trachea. This pressure should be limited to a range of $20-30 \mathrm{cms}$ of water to ensure adequate ventilation, prevent aspiration of secretions and guarantee a good tracheal perfusion ${ }^{1}$. During general anaesthesia the use of nitrous oxide and oxygen for ventilation can cause overdistention of the endotracheal tube cuff as nitrous oxide diffuses in the cuff 34 times faster than nitrogen diffuses out. As a result the increased intracuff pressure is exerted on the tracheal mucosa. This results in tracheal mucosal injury which can lead to postoperative sore throat, hoarseness of voice, tracheal ulceration and tracheal stenosis.

We compared the pressure changes in both the types of cuffs of the endotracheal tubes during general anaesthesia using nitrous oxide and oxygen. In one group the cuff was inflated with air and in the other group the cuff was inflated with nitrous oxide oxygen mixture aspirated from the breathing circuit.

\section{Materials and methods}

After institutional ethics committee approval and informed written consent 180 patients aged between 18-60 years, of both sexes, ASA status I \& II and posted for elective surgery of duration of approximately two hours were randomly allocated into 4 groups of 45 each. Patients requiring ventilator support in the 
postoperative period, patients undergoing head, face and neck surgeries, patients undergoing oral surgeries and patients with anticipated difficult airway were excluded from the study.

Group A1 - Portex high volume low pressure cuff tube used and cuff inflated with room air.

Group A2 -Portex high volume low pressure cuff tube used and cuff inflated with mixture of nitrous oxide and oxygen

Group B1 - Armoured low volume high pressure cuffed tube used and cuff inflated with room air.

Group B2 - Armoured low volume high pressure cuffed tube used and cuff inflated with mixture of nitrous oxide and oxygen.

General anaesthesia was given with endotracheal intubation in all the patients. After taking consent, confirmation of adequate starvation and premedication, standard monitors were attached and intravenous access was achieved. Patients were given Inj. Fentanylivas analgesic ( 2 microgm $/ \mathrm{kg}$ ) and Inj. Midazolam iv as sedative $(0.02 \mathrm{mg} / \mathrm{kg})$. Patient were induced with Inj. Propofol $2 \mathrm{mg} / \mathrm{kg}$ iv and inj. Vecuronium $0.01 \mathrm{mg} / \mathrm{kg}$ iv was used as muscle relaxant. After 3 mins of mask ventilation endotracheal intubation was done with Portex endotracheal tube in group A1 \& A2 and with armoured endotracheal tube in group B1 \& B2. The cuff of the endotracheal tube was inflated with either air or nitrous oxide- oxygenusing the minimal occlusive technique depending on the group. In the minimal occlusive techniquethe cuff is inflated slowly until no leak is heard during the peak airway pressure of the ventilator cycle. Anaesthesia was maintained with controlled ventilation with 60:40 nitrous oxide:oxygen and intermittent administration of inhalational agent sevoflurane. At the end of the surgery the patients were reversed with inj. Glycopyrrolateiv and inj. Neostigmine iv $(0.05 \mathrm{mg} / \mathrm{kg})$. Post operatively patients were observed for any complications such as sore throat, hoarseness of voice, stridor etc.

The sample of nitrous oxide and oxygen was collected in the following manner. A 20 ccs. syringe was attached to a three way stopcock. The other end of the three way was attached to the sampling port of the end tidal carbon dioxide sampling probe. This was attached in line on the circuit to the patient end of the breathing circuit. The sample was drawn by selectively turning the three way stopcock open to the syringe and the circuit's machine end. A20 ccs sample was drawn into the syringe. The sample was then locked in the syringe by turning the three way closed to the syringe.

Monitoring of intracuff pressure was done at every 30 minutes from the time the cuff was inflated to the time the patient was extubated. Patient was observed in the postoperative period for complications such as sorethroat, difficulty in swallowing, laryngitis etc. Statistical analysis was done by student ' $t$ ' test and chi square test.

\title{
III. Results
}

\section{Table 1}

\begin{tabular}{|l|l|l|l|l|}
\hline Group & Group A1 & Group A2 & Group B1 & Group B2 \\
\hline $\begin{array}{l}\text { Medium of cuff } \\
\text { inflation }\end{array}$ & Air & $\begin{array}{l}\text { Nitrous } \\
\text { oxygen }\end{array}$ & $\begin{array}{l}\text { Nitrous } \\
\text { oxygen }\end{array}$ \\
\hline Number of patients & $45 \mathrm{M}-23$ & $45 \mathrm{M}-23$ & Air & $45 \mathrm{M}-24$ \\
& F-22 & F-22 & F-21 & F-24 \\
\hline ASA status & I-42 & I-41 & I-40 & I-42 \\
& II-3 & II-4 & II-5 & II-3 \\
\hline
\end{tabular}

Table 1 shows that in our study all the groups were comparable with regards to age, gender and physical status.

\author{
M- males F-females
}

Table 2

Comparison of cuff pressures in Portex endotracheal tubes and armoured endotracheal tubes when air was used for cuff inflation

\begin{tabular}{|l|l|l|}
\hline Duration in minutes & Group A1 & Group B1 \\
\hline 0 & $30.08 \pm 15.40$ & $71.74 \pm 46.51$ \\
\hline 30 & $35.28 \pm 17.38$ & $79.45 \pm 48.23$ \\
\hline 60 & $41.17 \pm 20.96$ & $86.90 \pm 49.34$ \\
\hline 90 & $45.91 \pm 24.78$ & $96.57 \pm 49.77$ \\
\hline
\end{tabular}

By student $\mathrm{t}$ test $\mathrm{P}$ value $<0.05$ significant

Table 2 shows at 90 minutes the mean pressure in the cuff was significantly less in high volume low pressure group as compared to low volume high pressure group when air was used to inflate the cuff. 
Table 3

Comparison of cuff pressures in Portexendotracheal tubes and armoured endotracheal tubes when nitrous oxide and oxygen was used to inflate the cuff

\begin{tabular}{|l|l|l|}
\hline Duration in minutes & Group A2(cms of water) & Group B2(cms of water) \\
\hline 0 & $44.05 \pm 6.59$ & $83.65 \pm 37.78$ \\
\hline 30 & $43.05 \pm 7.07$ & $82.84 \pm 39.42$ \\
\hline 60 & $42.51 \pm 7.48$ & $82.65 \pm 38.21$ \\
\hline 90 & $44.13 \pm 7.50$ & $82.52 \pm 40.37$ \\
\hline
\end{tabular}

By student $t$ test $\mathrm{P}$ value $<0.05$ significant

Table 3 shows at 90 minutes the mean pressure in cuff was significantly less in high volume low pressure group as compared to low volume high pressure group when nitrous oxide and oxygen was used to inflate the cuff.

Table 4

Comparison of cuff pressures in Portexendotrachealtubes

By student $\mathrm{t}$ test

\begin{tabular}{|l|l|l|}
\multicolumn{2}{|c|}{ Comparison of cuff pressures in Portexendotrachealtubes } \\
\hline Duration in minutes & Group A1(cms of water) & Group A2(cms of water) \\
\hline 0 & $30.08 \pm 15.40$ & $44.05 \pm 6.59$ \\
\hline 30 & $35.28 \pm 17.38$ & $43.05 \pm 7.07$ \\
\hline 60 & $41.17 \pm 20.96$ & $42.55 \pm 7.48$ \\
\hline 90 & $45.91 \pm 24.78$ & $44.13 \pm 7.50$ \\
\hline
\end{tabular}

Table 4 shows at 90 minutes the mean pressure in Portex endotracheal tube cuff inflated with air went on rising over time as compared to when the cuff was inflated with nitrous oxide and oxygen.

Table 5

Comparison of cuff pressures in armoured endotracheal tubes

\begin{tabular}{|l|l|l|}
\hline Duration in minutes & Group B1(cms of water) & Group B2(cms of water) \\
\hline 0 & $71.74 \pm 46.51$ & $83.65 \pm 37.78$ \\
\hline 30 & $79.45 \pm 48.23$ & $82.84 \pm 39.42$ \\
\hline 60 & $86.90 \pm 49.34$ & $86.65 \pm 38.21$ \\
\hline 90 & $96.57 \pm 49.77$ & $82.52 \pm 40.37$ \\
\hline
\end{tabular}

By student t test

Table 5 shows at 90 minutes the mean pressure in armoured tube low volume high pressure cuff group steadily went on rising over time when air was used to inflate the cuff in comparison to when nitrous oxide and oxygen was used to inflate the cuff.

Table 6

Comparison of complications

\begin{tabular}{|c|c|c|c|c|c|c|c|c|}
\hline Complication & \multicolumn{2}{|c|}{ Group A1 } & \multicolumn{2}{|c|}{ Group B1 } & \multicolumn{2}{|c|}{ Group A2 } & \multicolumn{2}{|c|}{ Group B2 } \\
\hline & & $\%$ & No. & $\%$ & No. & $\%$ & No. & $\%$ \\
\hline Sore throat & 2 & 4.4 & 2 & 4.4 & 1 & 2.2 & 5 & 11.1 \\
\hline Difficulty in swallowing & 5 & 11.1 & 11 & 24.4 & 1 & 2.2 & 7 & $15.5^{*}$ \\
\hline Cough & 12 & 26.7 & 17 & 37.8 & 2 & 4.4 & 16 & $35.5^{*}$ \\
\hline Others & & - & & - & 1 & - & & -1 \\
\hline
\end{tabular}

By chi square test $* \mathrm{P}$ value $<0.05$ significant

Table 6 shows that difficulty in swallowing and cough were significantly more in the low volume high pressure cuff group even inspite of using nitrous oxide and oxygen to inflate the cuff as compared to high volume low pressure group.

\section{Discussion}

The inflatable cuff of an endotracheal tube provides both an airwayseal and protection from aspiration. However with time while using nitrous oxide and oxygen anaesthesia pressure inside the cuff increases. This pressure is exerted on the lateral wall of the trachea ${ }^{2}$. This results in mucosal damage, cartilaginous necrosis and eventual tracheal stenosis. All these are seen when endotracheal tube is used for prolonged period of time ${ }^{2,3,4,5}$. Revenas et $\mathrm{al}^{6}$ studied the pressure rise with time and how intracuff pressure rise was insignificant when nitrous oxide is used to inflate the cuffs. In our study we found the intracuff pressure rises with time, when air is used to inflate the cuff in both the groups. Pressure range over a period of time in all patients in high volume low pressure cuff was from $30.08 \pm 15.40$ to $45.91 \pm 24.78 \mathrm{cms}$ of water while that in low volume high pressure cuff was from $71.74 \pm 46.51$ to $96.57 \pm 49.77 \mathrm{cms}$ of water. When we used nitrous oxide- oxygen to inflate the cuff, the pressure in both the groups remained more or less steady. The range of pressure in high volume low pressure 
cuff was from $44.05 \pm 6.59$ to $44.13 \pm 7.50 \mathrm{cms}$ of water. In the armoured tube groups range was from $83.65 \pm 37.78$ to $82.52 \pm 40.37 \mathrm{cms}$ of water.

Revenaset $\mathrm{al}^{6}$ used nitrous oxide- oxygen mixture and concluded that there was no or minimal increase in intracuff pressure. Fischer et $\mathrm{al}^{7}$ were of the opinion that the problem of intracuff pressure can be tackled by inflating the cuff with $100 \%$ nitrous oxide instead of air. In this study the mean pressure in the high volume low pressure group were significantly lower than in low volume high pressure cuff group $(\mathrm{p}<0.05)$. Rate of increase in pressure was greater in cuffs inflated with air than when inflated with nitrous oxide oxygen mixture. In order to avoid cuff over inflation we inflated the cuff to minimal occluding volume. It has been observed in many studies and is also consistent with our findings that beyond a certain critical value, the addition of even a single $\mathrm{ml}$ of air results in a steep rise in intracuff pressure.

Expansion of air filled endotracheal tube cuffs during exposure to nitrous oxide in vitro is the result of diffusion of nitrous oxide into the cuff and slow diffusion of nitrogen out. Our data suggests that the same phenomenon occurs in cuffs in intubated patients. However cuff volume changes are less in vivo than in vitro. One reason for less diffusion of nitrous oxide in vivo is that less cuff surface area is available for diffusion. Only the inferior portion of the cuff, the portion not in contact with the tracheal wall is exposed to inspired gases in intubated patients. Other reason for less diffusion of nitrous oxide into the cuff in vivo than in vitro is higher initial and subsequent cuff pressure in the former resulting from tracheal wall restriction of cuff expansion. Increased cuff pressures must result in a proportional increase in the partial pressures of all the gases within the cuff. The latter tends to decrease the pressure gradient across the cuff wall for any nitrous oxide in the cuff and increase it for nitrogen both of which hinder cuff volume expansion.

P. J. Jenson et $\mathrm{al}^{8}$ studied the effect of tracheal tubes on the occurrence of sore throat in the postoperative period. The conclusion of the study was that low volume high pressure cuffs induced sore throat to a lesser extent than did high volume low pressure cuffed endotracheal tubes provided that the intracuff volumes were at the level of just seal volumes throughout the period of anaesthesia. When intracuff pressures in low volume high pressure cuffed tubes were high and allowed to increase, then this advantage was lost.

In our study we found that the low volume high pressure cuff group had a significantly higher incidence of complications than those in the high volume low pressure cuff group. This can be explained on the basis of the fact that these cuffs inflate eccentrically and are narrower than the high volume low pressure cuffs and are also made up of a thicker material whose mean thickness is $0.5436 \pm 0.59 \mathrm{~mm}^{9}$ thus these exert a more cuff tracheal wall pressure than the high volume low pressure cuff group.

From this study we conclude,

\section{Conclusion}

- The intracuff pressure in both the high volume low pressure cuff and the low volume high pressure cuff rises with time when the cuffs are inflated with air.

- The rise in intracuff pressure is not very significant when nitrous oxide- oxygen is used for inflating the cuffs in both the groups. The intracuff pressure in the low volume cuffs actually reduced over a period of time when nitrous oxide - oxygen was used for cuff inflation.

- The endotracheal tubes with smaller cuff size develop higher pressures in them than the larger diameter cuffs.

- The incidence of postoperative complications was higher in low volume high pressure cuffed tubes regardless of the inflating medium used as compared to high volume low pressure cuffed tubes.

References
[1]. Danielis M, et al, Continuous monitoring of endotracheal tube cuff pressure: best practice in intensive care unit, Assist Inferm Ric.,34(1), 2015, 15-20.

[2]. Leigh Julian, Maynard J.P., Pressure on the tracheal mucosa from cuffed tubes, British Medical Journal, 1979,1173-1174.

[3]. Carroll Robert, Hedden Michael, Safar Peter, Intratracheal cuffs: performance characteristics, Anaesthesiology, 31(3), 1969, 275281.

[4]. Mackenzie C. F., Klose S., Browne D. R. G., A study of inflatable cuffs on endotracheal tubes- pressures exerted on the trachea, British journal of Anaesthesia, 48, 1976, 105-109.

[5]. Keith King, MandavaBosobabu, Kamen Jack M., Tracheal tube cuffs and tracheal dilatation, Chest,67(4), 1975, 458-462.

[6]. Revenas and Lindholm C. E., Pressure and volume changes in tracheal tube cuffs during anaesthesia, Acta Anaesthesia Scandinivia, $20,1976,321-326$

[7]. Fischer Carl G., Cook D. Ryan, Endotracheal tube cuff pressure in the use of nitrous oxide, Anaesthesiology,73, 1991, 99.

[8]. Jenson P. J., Hommelgaard p., Sondergaard p., Erikson s., Sore throat after operation: influence of tracheal intubation, intracuff pressure and the type of cuff, British journal of anaesthesia, 54(4),1982, 453-457.

[9]. Bernhard William, Yost Leon, Turndorf Herman, Cottrel James et al, Physical characteristicsof and rates of nitrous oxide diffusion into tracheal tube cuffs, Anaesthesiology, 48(60, 1978, 413-417. 